\title{
O USO DE GAMIFICAÇÃO E DIFICULDADES MATEMÁTICAS: POSSÍVEIS APROXIMAÇÕES
}

João Coelho Neto - Programa de Pós-Graduação em Ensino - Universidade Estadual do Norte do Paraná - Campus de Cornélio Procópio - joaocoelho@uenp.edu.br

Marília Bazan Blanco - Programa de Pós-Graduação em Ensino - Universidade

Estadual do Norte do Paraná - Campus de Cornélio Procópio mariliabazan@uenp.edu.br

Juliano Aléssio da Silva - Programa de Pós-Graduação em Ensino - Universidade

Estadual do Norte do Paraná - Campus de Cornélio Procópio / UTFPR CP j.alessius@gmail.com

\section{RESUMO}

As tecnologias digitais em sala de aula podem ser instrumentos que auxiliam no processo de ensino e de aprendizagem, e para a área de educação especial, esses instrumentos podem ainda ser ferramentas potencializadoras de aprendizagem para indivíduos com deficiências ou transtornos, dentre eles, a Discalculia. Assim, este artigo visa identificar se há trabalhos que abordem o uso de gamificação em instrumentos para alunos com dificuldades de aprendizagem em Matemática e ou com Discalculia. O método utilizado nesta pesquisa foi a Revisão Sistemática de Literatura, em dois periódicos qualificados na área de Ensino (Qualis Periódicos - 2015), Revista Brasileira de Informática na Educação e a Revista de Novas Tecnologias na Educação, e nas seguintes base de dados: Scielo Library, BIREME Biblioteca, Science Direct, ACM Library e IEEE Xplore Digital Library. Como resultados, encontrou-se 2008 trabalhos, sendo 503 da área de Gamificação, três relacionados à Gamificação/Matemática e nenhum na área de Gamificação e dificuldades de ensino na Matemática ou Discalculia.

Palavras-chave: Gamificação. Matemática. Dificuldades de Ensino. Discalculia. Revisão Sistemática de Literatura.

\section{THE USE OF GAMIFICATION AND MATHEMATICAL DIFFICULTIES: POSSIBLE APPROACHES}

\begin{abstract}
Digital technologies in the classroom can be tools that aid in the teaching and learning process, and for the special education area, these tools can still be a potential learning tool for individuals with disabilities or disorders, such as Dyscalculia. Thus, this paper aims to identify if there are studies that address the use of gamification in instruments for students with learning difficulties in Mathematics or with Dysalculia. The method used in this research was the Systematic Literature Review, in two qualified periodicals in the Teaching Area (Qualis Periódicos - 2015), Revista Brasileira de Informática na Educação and the Revista de Novas Tecnologias na Educação and in the following databases: SciELO Library, BIREME Library, Science Direct, ACM Library e IEEE Xplore Digital Library. As results, we found 2008 papers, of which 503 of the Gamification area, three related to Gamification / Mathematics and none in the Gamification area and teaching difficulties in Mathematics or Dysalculia.
\end{abstract}

Keywords: Gamification. Mathematics. Difficulties in teaching. Dyscalculia. Systematic Literature Review. 


\section{Contextualização da Temática}

Dados da Prova Brasil apontam que $61 \%$ das crianças matriculadas no Ensino Fundamental brasileiro apresentam desempenho insatisfatório em Matemática (Qedu, 2017). Frente a dados como estes, as dificuldades de aprendizagem na Matemática, assim como o Transtorno de Aprendizagem da Matemática, a Discalculia, têm sido objetos de estudo de um número crescente de pesquisas.

Sabe-se que as dificuldades de aprendizagem na Matemática podem estar relacionadas a diversos fatores, desde alterações em circuitos neurais específicos, o que caracterizaria a Discalculia, até privações socioeconômicas e culturais e problemas pedagógicos, vinculados ao ensino da Matemática (Ciasca, 2004).

De acordo com Santos et al (2016), a Discalculia do desenvolvimento, também denominada de Transtorno específico de aprendizagem da aritmética, difere-se das dificuldades comuns na aprendizagem da Matemática de origem pedagógica, ambiental ou emocional, por relacionar-se a dificuldades mais elementares no senso numérico, como nomear e comparar números, e para realizar operações simples de adição e subtração; tem origem genética e envolve uma hipoperfusão (redução de fluxo sanguíneo) em áreas do córtex parietal. Embora de origem neurobiológica, sabe-se que o transtorno pode ser agravado por fatores pedagógicos e emocionais, como o ensino e a ansiedade à Matemática (Santos et al., 2010; Santos et al., 2016).

Dessa forma, as tecnologias digitais em sala de aula podem ser instrumentos que visam auxiliar no processo de ensino e de aprendizagem. Esses instrumentos podem também ser utilizados para alunos com necessidades educacionais especiais, visto que, em um ambiente escolar podem ser ferramentas motivadoras e transformadoras, pois segundo Braz et al. (2016, p. 757)

Um dos aspectos a serem considerados na transformação das escolas comuns brasileiras em instituições mais inclusivas é o trabalho com recursos e tecnologias contemporâneas. Isto porque tais recursos e tecnologias podem minimizar e até mesmo eliminar algumas barreiras que impedem pessoas com deficiência de conviver e de aprender com seus colegas e professores, conforme constroem a sua formação acadêmica e cidadã em escolas comuns.

Além disso, Sombrio, Schimmelpfeng e Ulbricht (2016) apontam que a inclusão de pessoas com necessidades educacionais especiais em escolas regulares precisa ser eficaz; portanto, métodos de ensino apropriados, materiais e professores capacitados são fatores indispensáveis para este contexto. Além disso, Brasil $(2009$, p.9) declara que "[...] o desenvolvimento de recursos e outros elementos de Tecnologia Assistiva têm propiciado a valorização, integração e inclusão dessas pessoas, promovendo seus direitos humanos".

Um dos meios para potencializar o uso das tecnologias no contexto educacional é a gamificação, que segundo Lopes, Toda e Brancher (2015), é um processo que consiste na utilização de elementos e mecânicas de jogos fora de seu escopo. Este processo vem sendo amplamente utilizado em diversas áreas, que vão desde a economia até o ensino, focando no treinamento e aprendizado a partir do engajamento dos indivíduos, Fardo (2013, p.1) também delineia que a “[...] gamificação vem ganhando visibilidade por sua 
capacidade de criar experiências significativas, quando aplicada em contextos da vida cotidiana".

Klock et al. (2014) corroboram que a gamificação está relacionada ao uso de elementos de jogos em contextos não relacionados com jogos, e que diferentes áreas fazem uso de sistemas gamificados, tais como entretenimento, saúde e especialmente a educação, uma vez que a gamificação fornece uma alternativa para engajar e motivar os estudantes durante o processo de aprendizagem (Klock et al., 2014; Ogawa et al., 2015). No entanto, para Dicheva et al. (2014,) embora a gamificação esteja ganhando espaço nas diversas áreas, como negócios, gestão empresarial e iniciativas de bem-estar, sua aplicação na educação é ainda uma tendência emergente e relativamente nova.

Com base nessas contextualizações, o objetivo do artigo é identificar se há instrumentos para alunos com dificuldades de aprendizagem em Matemática que utilizam a gamificação em seu contexto de desenvolvimento, este mapeamento dar-se-á por meio de uma revisão sistemática de literatura.

Esse artigo foi dividido em quatro seções: a primeira seção, contextualiza a temática foco dessa pesquisa; a segunda seção, descreve os materiais e métodos para a coleta e análise das informações; na terceira seção, as análises e discussões são apresentadas; na quarta e última seção, tecem-se as considerações finais acerca da temática e trabalhos futuros.

\section{Materiais e Métodos}

O método utilizado nesta pesquisa teve como base a proposta de Revisão Sistemática de Literatura apresentada por Kitchenham (2004), segundo a qual uma revisão objetiva identificar, avaliar e interpretar as pesquisas relevantes sobre determinada temática, a fim de responder um questionamento de pesquisa bem delimitado.

$\mathrm{Na}$ execução da presente revisão, elencou-se e adaptou-se as seguintes etapas de Kitchenham (2004):

1. Identificação e Planejamento da Pesquisa: a questão de pesquisa elencada neste trabalho têm como objetivo estruturar ações para a busca e interpretação dos resultados, sendo Q1: Há algum instrumento com diretrizes de gamificação para auxiliar alunos com dificuldades na Matemática ou Discalculia? Para contemplar a pergunta norteadora da pesquisa, os dados emergiram de diferentes bases de dados.

A primeira busca foi feita em dois periódicos qualificados (B2 - Revista Brasileira de Informática na Educação e B1 - Revista Novas Tecnologias na Educação) na área de Ensino (Classificação de Periódicos 2015), que publicam robustamente trabalhos relacionados ao uso das tecnologias digitais na educação. A seleção dos periódicos foi feita por meio do site Periódicos da Coordenação de Aperfeiçoamento de Pessoal de Nível Superior (CAPES) - Qualis 2015, com período de busca de 2006 a 2017, e feita entre março/abril de 2017. O critério de inclusão foi a identificação das palavras "Gamification" ou "Gamificação" nos títulos, para, a partir das pesquisas que apontavam a Gamificação em seu contexto, identificar trabalhos que vinculassem a Gamificação no desenvolvimento de instrumentos para o ensino da Matemática e, mais especificamente, 
trabalhos com ferramentas gamificadas para alunos com dificuldades na Matemática e/ou Discalculia.

Na segunda pesquisa, utilizou-se as seguintes base de dados: ScieELO Library e BIREME Biblioteca, com as palavras-chave "Gamification" e "Gamificação", como não foram identificados trabalhos com as referidas palavras-chave no título, não deu-se continuidade à busca com as palavras "Matemática" e "Mathematics". Nas bases Science Direct, ACM Library e IEEE Xplore Digital Library, foram feitas duas buscas, a primeira com a palavra-chave "Gamification" somente, e na segunda, com as palavras-chave "Gamification" and "Mathematics" em conjunto. A busca em algumas bases foi feita no idioma Inglês, visto que a maioria dos trabalhos são publicados neste idioma.

2. Seleção de estudos primários: para a seleção dos trabalhos pertinentes, identificou-se os que apresentavam a palavra-chave "Gamificação", "Gamification" e a combinação no título "Gamification" and "Mathematics", seguida da leitura dos abstracts, descartando-se os artigos que não tratavam da temática.

3. Estudo da avaliação de qualidade.

4. Extração e monitorização de dados: nesta etapa foram realizadas leitura e análise dos trabalhos selecionados, assegurando a pertinência desses em relação à temática pesquisada.

5. Síntese de dados: a partir da identificação dos trabalhos relevantes, fez-se uma síntese de seus resultados, que seguem expostos na seção a seguir.

\section{Resultados e Discussão}

A primeira pesquisa foi feita em duas revistas que tratam do uso da informática na educação, com a palavra-chave "Gamificação" ou "Gamification". Os resultados dessa busca podem ser apreciados na Tabela 1, que apresenta o mapeamento dos trabalhos na Revista Brasileira de Informática na Educação- RBIE, e na Tabela 2, que descreve os resultados obtidos na Revista de Novas Tecnologias na Educação - RENOTE

Tabela 1- Revista Brasileira de Informática na Educação - RBIE

\begin{tabular}{l|l|l|l|l|l}
\hline ANO & $\begin{array}{l}\text { Volume / } \\
\text { Número }\end{array}$ & Quantidade & $\begin{array}{l}\text { Quantidade } \\
\text { Gamificação }\end{array}$ & $\begin{array}{l}\text { Quantidade } \\
\text { Gamificação } \\
\text { Matemática }\end{array}$ & $\begin{array}{l}\text { Quantidade } \\
\text { Gamificação } \\
\text { Matemática - } \\
\text { Dificuldades } \\
\text { Aprendizagem }\end{array}$ \\
\hline $\mathbf{2 0 0 6}$ a & $\begin{array}{l}\text { Vol. 14/1 a } \\
\mathbf{2 0 1 4}\end{array}$ & 188 & 0 & 0 & 0 \\
\hline $\mathbf{2 0 1 5}$ & Vol. 22/3 $23 / 1$ & 16 & 0 & 0 & 0 \\
\hline & Vol. 23/2 & 15 & 0 & 0 & 0 \\
\hline & Vol. 23 / 3 & 13 & 1 & 0 & 0 \\
\hline $\mathbf{2 0 1 6}$ & Vol. 24/1 & 12 & 1 & 0 & 0 \\
\hline & Vol. 24 / 2 & 11 & 1 & 0 & 0 \\
\hline & Vol.24/3 & 10 & 0 & 0 & 0 \\
\hline & & $\mathbf{2 6 5}$ & $\mathbf{3}$ & $\mathbf{0}$ & $\mathbf{0}$ \\
\hline
\end{tabular}

Fonte: Os Autores 
Na RBIE, dos 265 trabalhos dos últimos 10 anos, três trabalhos tratavam da temática de Gamificação, mas nenhum deles referente à Gamificação / Matemática / Dificuldades de Aprendizagem e nem sobre Discalculia. Desse modo, não houve análise dos mesmos no presente artigo.

A segunda análise iniciou-se na Revista de Novas Tecnologias na Educação RENOTE, visto esta ser de grande influência na área de Informática na Educação no Brasil, por publicar diferentes abordagens do uso das tecnologias digitais da informação e comunicação para o contexto escolar. A Tabela 2 mostra uma síntese dos resultados da pesquisa.

Tabela 2 - Revista de Novas Tecnologias - RENOTE

\begin{tabular}{|c|c|c|c|c|c|}
\hline ANO & $\begin{array}{l}\text { Volume / } \\
\text { Número }\end{array}$ & Quantidade & $\begin{array}{l}\text { Quantidade } \\
\text { Gamificação }\end{array}$ & $\begin{array}{l}\text { Quantidade } \\
\text { Gamificação } \\
\text { Matemática }\end{array}$ & $\begin{array}{l}\text { Quantidade } \\
\text { Gamificação } \\
\text { Matemática - } \\
\text { Dificuldades } \\
\text { Aprendizagem }\end{array}$ \\
\hline $\begin{array}{l}2006 \text { a } \\
2012\end{array}$ & $\begin{array}{l}\text { Vol. 4/1 a } \\
\text { Vol. 10/3 }\end{array}$ & 785 & 0 & 0 & 0 \\
\hline \multirow[t]{3}{*}{2013} & Vol.11/1 & 63 & 1 & 0 & 0 \\
\hline & $\begin{array}{l}\text { Vol. } 11 \text { / } 2 \text { - } \\
\text { Especial } \\
\text { (PEAD) }\end{array}$ & 16 & 0 & 0 & 0 \\
\hline & Vol.11/3 & 65 & 0 & 0 & 0 \\
\hline \multirow[t]{2}{*}{2014} & Vol. 12/1 & 51 & 0 & 0 & 0 \\
\hline & Vol. 12 / 2 & 59 & 3 & 0 & 0 \\
\hline \multirow[t]{2}{*}{2015} & Vol. 13 / 1 & 46 & 1 & 0 & 0 \\
\hline & Vol. 13/2 & 43 & 2 & 0 & 0 \\
\hline \multirow[t]{3}{*}{2016} & Vol. 14 / 1 & 56 & 1 & 0 & 0 \\
\hline & Vol. 14 / 2 & 65 & 1 & 0 & 0 \\
\hline & & 1249 & 9 & $\mathbf{0}$ & $\mathbf{0}$ \\
\hline
\end{tabular}

Fonte: Os autores

Na RENOTE, dos 1249 trabalhos dos últimos 10 anos, nove trabalhos tratavam da temática de Gamificação, mas nenhum referente à Gamificação / Matemática / Dificuldades de Aprendizagem e nenhuma abordagem para a Discalculia. Assim, mais uma vez, a análise dos artigos não foi realizada, já que não abordavam o tema de interesse da pesquisa.

Desse modo, nesta primeira busca, dos 1514 trabalhos mapeados nas revistas RBIE e RENOTE, somente 12 artigos trabalhavam da temática de Gamificação e conforme já apontado, nenhum sobre Matemática e ou Dificuldades de Aprendizagem na Matemática e/ou Discalculia.

Para a segunda pesquisa, utilizou-se as seguintes base de dados: SciELO Library e Bireme Biblioteca, com as palavras-chave "Gamification" ou "Gamificação", e nas bases Science Direct, ACM Library e IEEE Xplore Digital Library, com a palavra-chave "Gamificação" somente ou com as palavras-chave "Gamification" and "Mathematics" em conjunto. Os resultados podem ser observados na Tabela 3. 
Tabela 3 - Busca e Resultado das Bases de Dados

\begin{tabular}{|c|c|c|c|c|}
\hline Base de Dados & Intervalo & Busca & Palavras-chave & Quantidade \\
\hline SciELO Library & Não Há & Título & "Gamificacão" & 0 \\
\hline SciELO Library & Não Há & Título & "Gamification" & 0 \\
\hline $\begin{array}{l}\text { Biblioteca } \\
\text { Bireme }\end{array}$ & Não Há & Título & "Gamificação" & 0 \\
\hline $\begin{array}{l}\text { Biblioteca } \\
\text { Bireme }\end{array}$ & Não Há & Título & "Gamification" & 0 \\
\hline Science Direct & 2006 a 2017 & Título / journals & "Gamification" & 83 \\
\hline Science Direct & $2016-2017$ & Título / journals & $\begin{array}{l}\text { "Gamification" and } \\
\text { "Mathematics" }\end{array}$ & 0 \\
\hline ACM Library & Não Há & $\begin{array}{c}\text { Título / matches } \\
\text { all }\end{array}$ & "Gamification" & 193 \\
\hline ACM Library & Não Há & $\begin{array}{c}\text { Título / matches } \\
\text { all }\end{array}$ & $\begin{array}{l}\text { "Gamification" and } \\
\text { "Mathematics" }\end{array}$ & 2 \\
\hline $\begin{array}{l}\text { IEEE Xplore } \\
\text { Digital Library }\end{array}$ & $\begin{array}{l}2006- \\
\text { Present }\end{array}$ & $\begin{array}{c}\text { Título / Document } \\
\text { Title / Conference } \\
\text { Publications }\end{array}$ & "Gamification" & 215 \\
\hline $\begin{array}{l}\text { IEEE Xplore } \\
\text { Digital Library }\end{array}$ & $\begin{array}{l}2006- \\
\text { Present }\end{array}$ & $\begin{array}{l}\text { Título / Document } \\
\text { Title / Conference } \\
\text { Publications }\end{array}$ & $\begin{array}{l}\text { "Gamification" and } \\
\text { "Mathematics" }\end{array}$ & 1 \\
\hline
\end{tabular}

Fonte: Os autores

$\mathrm{Na}$ base de dados ACM Library, encontrou-se dois artigos que combinavam as palavras-chave "Gamification" and "Mathematics" dos autores (Kachurina et. al., 2015 e Flores; Ramírez, Mena, 2016). Kachurina et al. (2015) apresentam que o foco da pesquisa é feito sobre a gamificação e Modelagem Matemática, visto que as junções dessas tendências podem ter valiosas contribuições para programas governamentais de melhoria da saúde da população; enquanto Flores; Ramírez, Mena (2016) discutem como a estratégia de gamificação baseada em desafios contribui para a aprendizagem significativa do cálculo de sólidos. A avaliação do desempenho dos alunos durante as atividades de gamificação seguiu o ciclo de Modelagem Matemática.

Na base de dados IEEE Xplore Digital Library, identificou-se um artigo que combinava as palavras-chave "Gamificação" e "Matemática", dos autores Lameras e Moumoutzis (2015), o qual investiga o uso da gamificação com uma abordagem de sala invertida e aprendizagem significativa, referente aos conhecimentos matemáticos, por meio de um arcabouço denominado GamifyMaths, de modo a propiciar aos alunos e professores a conexão da Matemática com outras disciplinas.

Dessa forma, vislumbrou-se que nenhum dos artigos supracitados trabalha a relação da inserção da gamificação no desenvolvimento de produtos/games para alunos com dificuldades de aprendizagem em Matemática e/ou Discalculia. 


\section{Considerações Finais}

Este artigo visou mapear, à luz das etapas da Revisão Sistemática de Literatura, instrumentos para alunos com dificuldades de aprendizagem em Matemática e/ou Discalculia que utilizam a gamificação em seu contexto de desenvolvimento. Para tanto, a questão norteadora da revisão foi Q1: Há algum instrumento com diretrizes de gamificação para auxiliar alunos com dificuldades na Matemática e/ou Discalculia?

Com o mapeamento realizado, vislumbrou-se que, na primeira busca 1514 trabalhos foram verificados, sendo que somente 12 abordaram a área de Gamificação e nenhum da área de Gamificação/Matemática, quiçá, na área de dificuldades de Matemática e/ou Discalculia. A partir desse número relativamente baixo, entende-se que esta área ainda é nova, conforme aponta Dicheva et al. (2014).

Para a segunda busca, retornaram 494 trabalhos com o termo de busca "Gamificação", "Gamification e "“Gamification" and "Mathematics"”, retornaram somente três trabalhos, nos quais não identificou-se relação entre Gamificação e Dificuldades de Aprendizagem na Matemática e/ou Discalculia. Assim, respondendo a Q1 desta revisão, verificamos que não houve trabalhos que permearam a temática foco deste estudo, que foi a de verificar a existência de ferramentas relacionadas com a Gamificação e Dificuldades de Aprendizagem ou Discalculia.

Desse modo, vislumbrou-se que dos 2008 artigos mapeados e analisados, nenhum tratou da temática foco dessa pesquisa, assim, identifica-se a necessidade de pesquisas sobre esta temática, já que as dificuldades de aprendizagem na Matemática são frequentes em sala de aula, e a gamificação tem-se mostrado uma ferramenta promissora nos ambientes de ensino e aprendizagem em todos os níveis de ensino.

\section{Referências}

ARNOLD, BRIAN J. Gamification in Education. In: ASBBS Proceedings; San Diego. San Diego: American Society of Business and Behavioral Sciences. (Feb 2014), 21.1, p.32-39.

BRASIL. Subsecretaria Nacional de Promoção dos Direitos da Pessoa com Deficiência. Comitê de Ajudas Técnicas. Tecnologia Assistiva. - Brasília: CORDE, 2009. 138 p.

BRAZ, L. M.; RAMOS, E.S.; BENEDETTI, M.L.P.; HORNUNG, H.H. Design de tecnologia e educação inclusiva: explorando o espaço do problema. In: V Congresso Brasileiro de Informática na Educação (CBIE2016), anais do XXVII Simpósio Brasileiro de Informática na Educação (SBIE 2016), Uberlândia, Minas Gerais. Anais, 2016, p. 757-766.

CIASCA, S.M. Distúrbios de aprendizagem- uma questão de nomenclatura. Revista Sinpro, Edição Especial Dificuldades de Aprendizagem, p.4-8, 2004. Disponível em: http://www.sinpro-rio.org.br/download/revista/revistadificuldades.pdf. Acesso em: 20 abr. 2017. 
DICHEVA, D.; DICHEV, C.; AGRE, G. \& ANGELOVA, G. Gamification in Education: A Sytematic Mapping Study. Educational Tecnology \& Society, 18(3), p. 75-88, 2014.

FARDO, M.L. A gamificação aplicada em Ambientes de Aprendizagem. Revista Novas Tecnologias na Educação - RENOTE. V. 11 No 1, p. 1-9, julho, 2013.

FLORES, E.G.; MONTOYA, M.S.R.; MENA, J. Challenge-based gamification and its impact in teaching mathematical modeling. In: TEEM '16: Proceedings of the Fourth International Conference on Technological Ecosystems for Enhancing Multiculturality. Salamanca, Spain - November 02 - 04, 2016, p. 771-776.

KACHURINA, P.; KOLOMIYTSEV, S.; BESPALOV, A.; BARCHUK, A.; PANTYUKHOV, P. The Synergy of Gamification and Mathematical Modelling in eHealthcare. In: EGOSE '15: Proceedings of the 2015 2nd International Conference on Electronic Governance and Open Society: Challenges in Eurasia, November 2015, p.116-122.

KITCHENHAM, B. A. Procedures for Performing Systematic Reviews. Tech. Report TR/SE-0401, Keele University, 2014.

KLOCK, A.C.T.; CARVALHO, M.F.; ROSA, B.E.; GASPARINI, I. Análise das técnicas de gamificação em ambientes virtuais de aprendizagem. Revista Novas Tecnologias na Educação - RENOTE. V. 12 No 2, dezembro, p.1-10, 2014.

LAMERAS, P.; MOUMOUTZIS, N. Towards the Gamification of Inquiry-Based Flipped Teaching of Mathematics: A Conceptual Analysis and Framework. In: 2015 International Conference on Interactive Mobile Communication Technologies and Learning (IMCL): 2015, p. 343-347.

LOPES, R.A.; TODA, A.M.; BRANCHER, J.D. A preliminary study about gamification intrinsic and extrinsic motivators in single and multiuser environments. Revista Brasileira de Informática na Educação. Volume 23, Número 3, p. 164-173, 2015.

OGAWA, A.N.; MAGALHÃES, G.G.; KLOCK, A.C.T.; GASPARINI, I. Análise sobre a gamificação em ambientes educacionais. Revista Novas Tecnologias na Educação RENOTE. V. 13 No 2, dezembro, p.1-10, 2015.

QEDU. Prova Brasil. 2017. Disponível em: http://www.qedu.org.br/. Acesso em: 20 abr. 2017.

SANTOS, F.H.; RIBEIRO, F.S; KIKICHI, R.S.; SILVA, P.A. Recomendações para professores sobre o Transtorno da Matemática. Revista Sinpro-Rio, No 05, maio, p.1833, 2010.

SANTOS, F. H. dos, et al. Cognição Numérica: Contribuições à Pesquisa Clínica. In: PRADO, P. S. T. do, CARMO, J. dos S. (Orgs.). Diálogos sobre ensino-aprendizagem 
da matemática. Abordagens pedagógica e neuropsicológica. São Paulo: Cultura Acadêmica, 2016, p.63-91.

SOMBRIO, G. S.; SCHIMMELPFENG, L. E.; ULBRICHT, V. R. The production of a gamified Learning Object accessible to people with visual or hearing disabilities for teaching Geometry. In: Latin American Conference on Learning Objects and Technology (LACLO), 3-7 Oct. 2016. 